\title{
UN EXPERIENCIA EDUCATIVA TELEVISADA EN ESPAÑA ENTRE LA DÉCADA DE LOS 60 Y LOS 80. EL PROGRAMA MISIÓN RESCATE
}

\section{An educational experience televised in Spain between the 60's and 80's. The Mission Rescue program}

\author{
M. ${ }^{a}$ Cristina Hernández Castelló \\ Universidad de Valladolid \\ Correo-e: mariacristina.hernandez@uva.es
}

Recepción: 3 de mayo de 20I8. Envío a revisores: 8 de mayo de 2018 Aceptación definitiva: 20 de julio de 2018

RESUMEN: Este texto tiene como propósito fundamental visualizar la función educativa que en las décadas de los 60, 70 y 80 tuvo en España el programa Misión Rescate en relación con el patrimonio cultural material. Exponiendo para una mejor comprensión de su importancia un caso concreto, el redescubrimiento de los mosaicos romanos y la necrópolis visigoda de Santa Lucía por el grupo de rescate n. ${ }^{\circ}$ 349 de la Escuela Graduada de Aguilafuente (Segovia).

Palabras clave: educación patrimonial; didáctica de la historia del Arte; didáctica de las Ciencias Sociales; Aguilafuente (Segovia); Misión Rescate.

AвsтRAcт: This text has as its fundamental purpose visualizes the educational function that in the decades of the 60, 70 and 80 had in Spain the program «Mission Rescue» in relation to the heritage cultural material. Exposing for a better understanding of its importance, a concrete case, the rediscovery of the Roman mosaics and the Visigothic necropolis of Santa Lucia by the rescue group n. 349 of the Graduate School of Aguilafuente (Segovia).

KEY WORDs: heritage education; didactic of Art history; didactic of Social Sciences; Aguilafuente (Segovia); Mission Rescue. 


\section{Introducción}

$\mathrm{P}$ RETENDEMOS REVALORIZAR EL PAPEL FUNDAMENTAL que jugaron en la salvaguarda y redescubrimiento del patrimonio material español miles de jóvenes que participaron en el programa de Radio Nacional de España y Radio Televisión Española Misión Rescate. Mediante la búsqueda, hallazgo y revalorización de bienes artísticos y arqueológicos a lo largo y ancho de la Península acapararon la atención mediática sobre cientos de obras de arte y restos arqueológicos. A su vez pretendemos constatar cómo este proyecto de educación informal para niños y jóvenes llegó a implicar a toda la comunidad local en cada lugar donde existió un grupo concursando en el programa.

Del mismo modo, buscamos evidenciar la importancia que tuvieron ya por entonces la radio y la televisión como vehículos efectivos de educación informal para la puesta en valor del patrimonio a través de un programa dirigido a los miembros más jóvenes de la sociedad. Albergamos la esperanza de servir como acicate en el siglo xxi para una mayor apuesta sobre el papel del mundo audiovisual en la divulgación, conservación y salvaguarda del patrimonio. Esperando que el conocimiento de las antiguas prácticas sobre educación patrimonial pueda reverter en un aprovechamiento de estas mediando la obviamente necesaria adaptación a los tiempos actuales.

Como ejemplo para ilustrar los puntos antes referidos hemos revisado un caso concreto que llamó nuestra atención por la importancia que tuvieron los hallazgos realizados por un grupo de jóvenes de la localidad de Aguilafuente (Segovia), así como por el efecto que estos descubrimientos tuvieron en su entorno con el

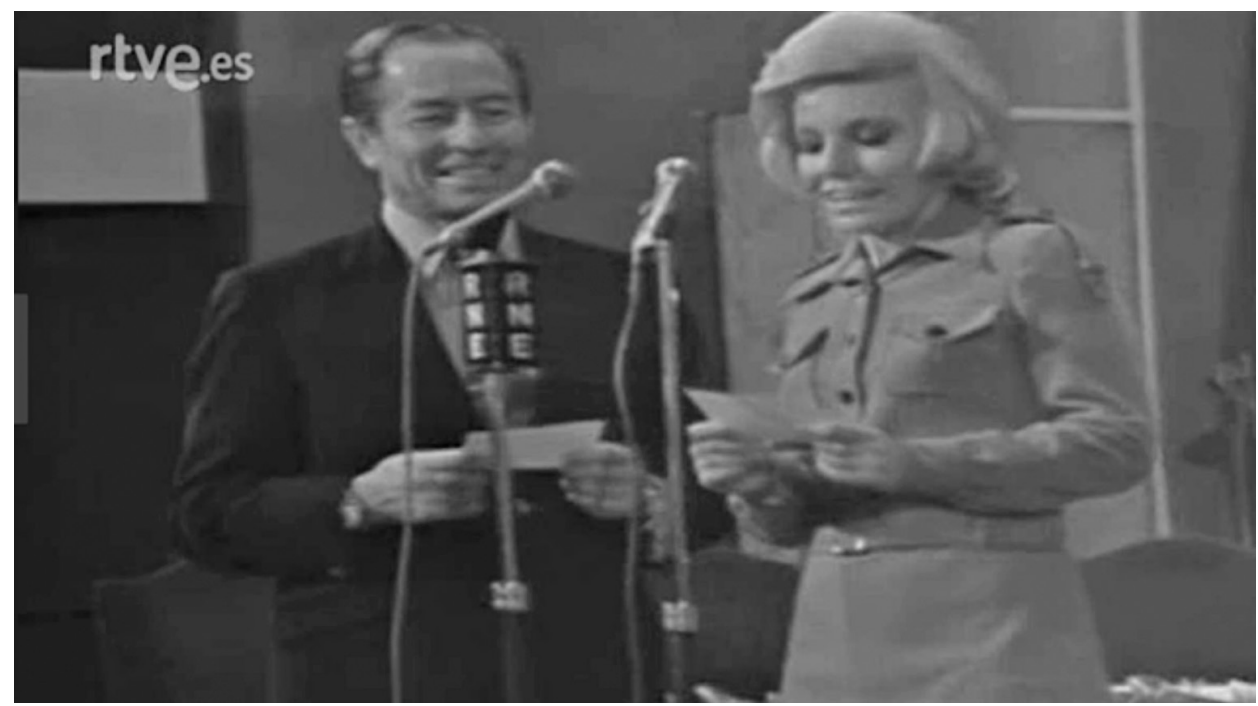

I. Kiko Ledgard y María Luisa Seco presentan el I2 de octubre de i97I el programa especial de entrega de premios de Misión Rescate. Fuente: Archivo Radio Televisión Española. 
paso del tiempo. Las pesquisas llevadas a cabo por un grupo de alumnos de la Escuela Graduada de aquella localidad en el denominado paraje de San Lucía, a solo dos kilómetros escasos hacia el sudeste del centro urbano, dieron como resultado el redescubrimiento de uno de los yacimientos más interesantes de la provincia tanto por la calidad de los restos hallados como por la estratigrafía arqueológica que presenta el sitio, formada por restos de una villa de época romana fechada en torno al siglo iv d. C., una necrópolis visigoda del siglo vi d. C. y por los vestigios de un ermita medieval dedicada a Santa Lucía que dio nombre al lugar ${ }^{1}$.

Como fuentes documentales para este trabajo hemos utilizado las noticias que sobre el programa Misión Rescate fueron publicadas en prensa en los años de su emisión, fundamentalmente en el $A B C$, El País y La Vanguardia, centrándonos para obtener información sobre el yacimiento de Aguilafuente en la hemeroteca del primer periódico de Castilla y León, El Norte de Castilla.

\section{Misión Rescate: la educación en los medios}

A finales de la década de los 60 el jefe de programas escolares de Radio Nacional de España (RNE) Aníbal Arias creó y promovió Misión Rescate, con la participación de RNE y la estrecha colaboración de la Dirección General de Bellas Artes. Posteriormente se unió a este proyecto Televisión Española (TVE) produciéndose un salto importante en la difusión del mismo gracias al paso de las ondas a la televisión.

El programa obtuvo un gran éxito manteniéndose en parrilla is años -del is de octubre de 1967 hasta los inicios de $1982^{2}-$, convirtiéndose en uno de los espacios más longevos de la época dedicado al público infantil y juvenil's, si bien, como señala Tamara Antonio Jimeno en su tesis doctoral, su radio de acción superaba la franja de edad mencionada pudiendo catalogarse como un programa dedicado al «público familiar» ${ }^{4}$.

Este espacio de educación patrimonial fue configurado a la manera de un concurso en el que grupos de alumnos de todo el país dirigidos por un profesor competían por localizar, descubrir o actualizar una obra de arte o vestigio histórico que se hubiera mantenido ignorado, poco conocido o infravalorado en su entorno.

En el 20or Isaac Sastre de Diego afirmaba que aún queda «algo menos de la mitad de la villa por descubrir», en SASTre de Diego, I.: «La villa romana de Santa Lucía (Aguilafuente, Segovia). Aproximación a su estudio treinta años después de su excavación», Espacio, Tiempo y Forma, Serie I, Prehistoria y Arqueología, t. I4 (200I), p. 290.

$2 A B C$, Sevilla o9/oi/1982, p. 28.

3 Paz Rebollo, María Antonia y Martínez Valero, Lizette: «La primera conformación de una audiencia infantil y juvenil para la televisión en España (1958-1968)», Estudios sobre el mensaje periodístico, 20, número especial (20I4), p. 58. Sitúan los autores el fin de la presencia de este programa en parrilla en noviembre de 1978, sin embargo, en la sección Cartas al director del periódico $A B C$ o9/or/I982, p. 2, Martín Recio Vengazones afirmaba, «acaba de morir un programa popular educativo: "Misión Rescate"».

4 Antonio Jimeno, Tamara: tesis doctoral, Madrid, 20i6, p. I6I. 


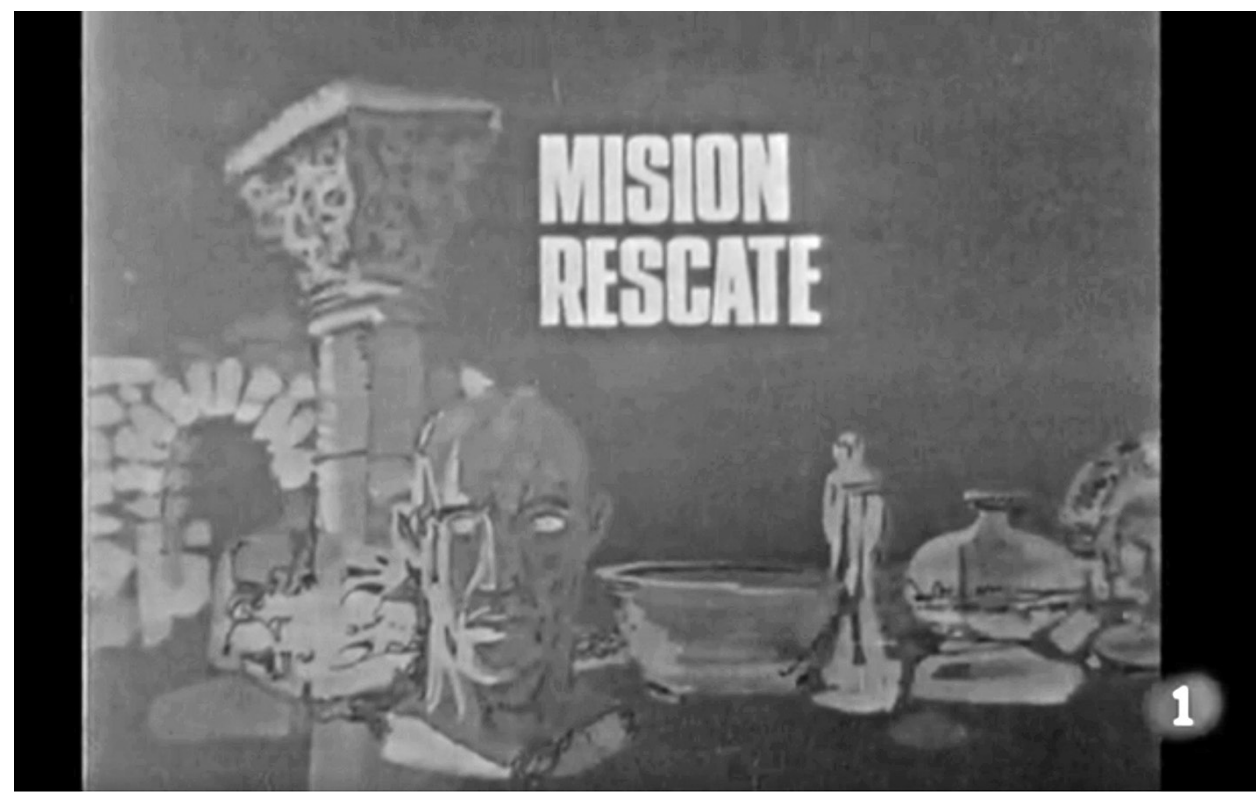

2. Cabecera del programa en RTVE. Fuente: Archivo Radio Televisión Española.

La motivación y el interés entre los jóvenes concursantes surgía, por tanto, de su conexión directa con el entorno, de su vínculo personal con los proyectos de rescate propuestos por sus maestros, convirtiéndose en proyectos significativos a través de la vinculación entre patrimonio y emoción.

Tan solo dos años después de su puesta en marcha, el maestro del Centro de Orientación y Documentación. Didáctica de Enseñanza (CEDodeP)s Armando Fernández Benito explicaba que el objetivo principal del programa era entusiasmar a los jóvenes con una empresa motivadora, utilizando una nomenclatura que sintonizaba con el espíritu de aventura y con la psicología de las edades a las que estaba dirigido el concurso ${ }^{6}$.

Se articulaba a pequeña escala como una multitud de microproyectos de carácter lúdico, motivador y formativo llevados a cabo por grupos de niños y adolescentes que diseminados por toda la geografía española contribuían a una causa mayor, la puesta en valor y salvaguarda del patrimonio nacional, mientras competían por alcanzar uno de los ansiados trofeos que el programa otorgaba. Los principales premiados, aquellos que alcanzaban los objetivos más importantes, obtenían los trofeos de oro y plata. También existían otros premios de menor categoría para objetivos artísticos, históricos y arqueológicos que habían destacado. Asimismo, se entregaban un número variable de menciones especiales. Los principales

El Centro de Orientación y Documentación. Didáctica de Enseñanza Primaria (CEdodep), fue creado en 1958 .

6 Fernández Benito, Armando: «Convocatoria en las Ondas. Misión Rescate», Vida escolar, Madrid, n. ${ }^{\circ}$ IO6-IO7 (I969), p. 46. En ese momento 438 grupos de alumnos participaban en el concurso. 
galardones llevaban aparejados premios en metálico que iban desde las 25.000 a las 50.000 pesetas, cuantía que subió hasta las ioo.000 pesetas en las últimas ediciones del concurso ${ }^{7}$, incluso algunos premiados con el trofeo de oro disfrutaron de viajes culturales por España ${ }^{8}$. Todos estos premios tenían otra recompensa, la posibilidad de aparecer en Televisión Española con un pequeño reportaje sobre la misión emprendida. Esos minutos en la pantalla contribuían a publicitar a nivel nacional aquellas localidades premiadas mejorando el porvenir turístico de la zona9.

Tal fue el éxito del programa que en la entrega de premios y galardones de cada campaña participaban personalidades destacadas del ámbito político y cultural del momento.

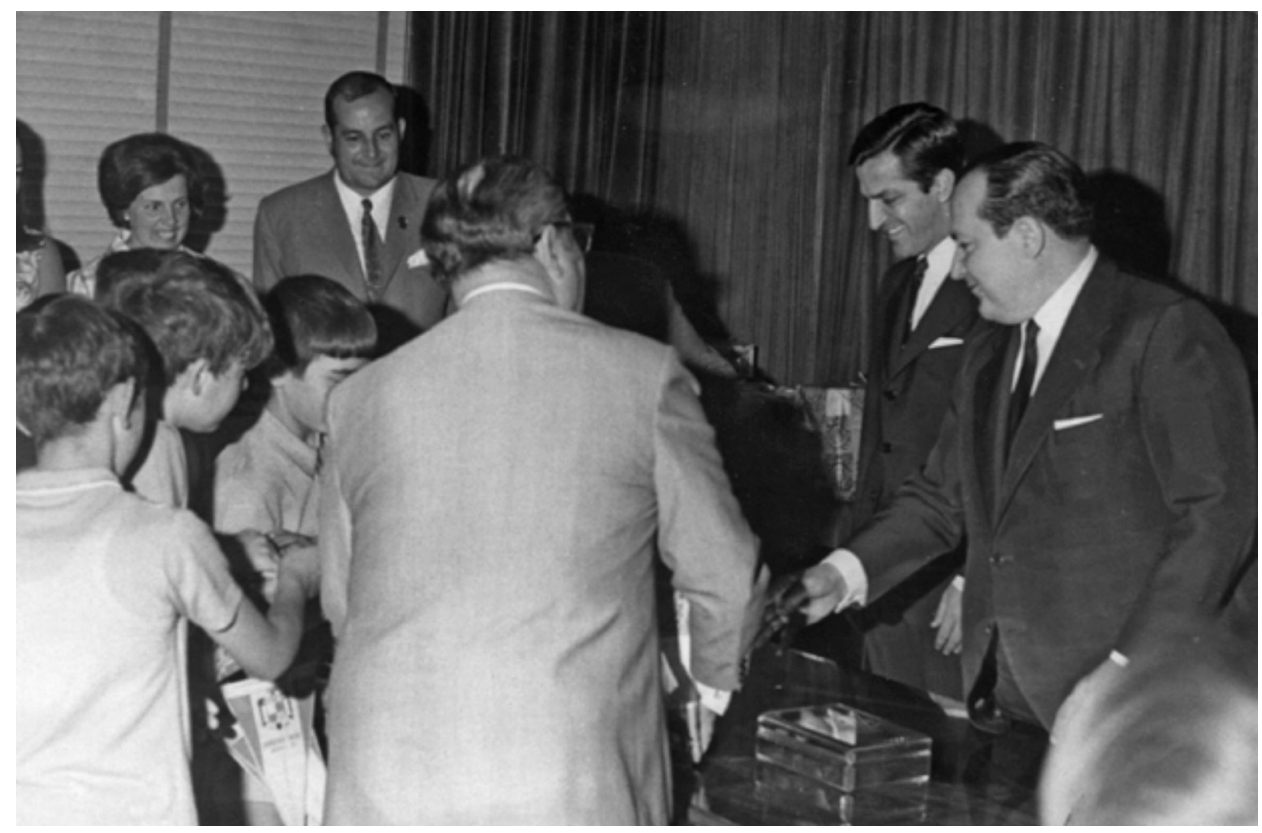

3. El grupo 208 de Ubrique (Cádiz), capitaneados por su maestro Manuel Cabello, reciben uno de los galardones de manos del ministro Sánchez Bella y de Adolfo Suárez ${ }^{10}$. Archivo fotográfico: familia Cabello Izquierdo ${ }^{\text {II }}$.

El País, I5/II/198I en https://elpais.com/diario/r981/1I/15/radiotv/374626802_850215.html (consultado el I5/12/2017).

$8 A B C$, Edición de Andalucía, Io/ro/r969.

- La Corporación Municipal de Ubrique (Cádiz) de donde procedía uno de los grupos más activos en Misión Rescate, dedicaron una plaza al programa que hoy en día se mantiene en el callejero.

1o Entre 1969 y 1973 Adolfo Suárez fue director de Radio Televisión Española. Creó un consejo asesor para diseñar la programación de parrilla en el que incluyó a periodistas y profesores universitarios, sin embargo, y como era de esperar en el contexto de la época, también contó entre sus asesores con militares y eclesiásticos.

" Agradecemos a la familia Cabello Izquierdo el habernos proporcionado material fotográfico para ilustrar estas páginas. 
El concurso fue organizado como si de una gran empresa militar se tratase, algo que no era de extrañar en los últimos años del franquismo ${ }^{12}$. Así lo resumía el maestro del CEDODEP:

La estrategia de una campaña en campos de surcos y paz, cual son la Historia y el Arte, se plantea a través de un Estado Mayor conjunto (Radio Nacional de España y Televisión Española, con la colaboración de la Dirección General de Bellas Artes); unidades de acción, con sus mandos (Grupos, Comandos, Patrullas y Batidores de Rescate), que buscan, localizan, rescatan y estudian una obra artística o histórica de su localidad (Objetivo). Alcanzado el objetivo, tras la satisfacción y el honor de la "Misión Cumplida", el mando puede conceder, a la vista de la importancia del hallazgo, las oportunas recompensas (Trofeos de Oro, Trofeo de Plata o Mención de Honor ${ }^{13}$.

En las bases para participar se establecía que todos los colegios de centros públicos y privados de EGB y FP del país podían inscribir a sus Grupos de Rescate. Estos debían estar dirigidos por un maestro de la institución y formados por un máximo de cinco alumnos en edades comprendidas entre los 9 y los iz años, rango de edad que aumentó a mediada que el concurso avanzaba llegando hasta los i6 años en $1980^{14}$. Los Grupos de Rescate debían emprender sus misiones fuera del horario escolar ${ }^{15}$, siguiendo la modalidad de educación informal, pues la actividad debía desarrollarse fuera del horario escolar. El nombre que correspondía a cada uno estaba formado por el nombre del centro al que representasen y el número de orden que les correspondiese y les fuese asignado tras la inscripción.

Al limitar el número de niños que podían formar parte de los Grupos de Rescate, la actividad quedaba relegada a unos pocos, por lo que se adoptó una solución que aumentaba la ratio de participantes en cada campaña, de manera que cada centro educativo inscrito podía organizar otro tipo de grupos con niños y jóvenes de su localidad. Por un lado, existían los Comandos de Rescate, que eran dirigidos por un catedrático o profesor de enseñanza media. Además, existía la posibilidad de crear un grupo auxiliar, el denominado Cuerpo de Batidores, al que podían pertenecer alumnos de enseñanza primaria o bachillerato, con independencia de que en su propio centro existiese ya un Grupo o Comando de Rescate organizado. Los batidores se inscribían de forma individual y se les otorgaba una credencial. En el anverso de la credencial se incluía la siguiente nota: «Se ruega a cuantas personas amantes del arte sea presentada esta CREDENCIAL,

12 Para comprender el papel educativo de la televisión en la época cabe destacar los siguientes estudios: Paz Rebollo, María Antonia y Martínez Valerio, Lizette: «Nuevos programas para nuevas realidades: la programación infantil y juvenil en TVE (1969-1975)», Journal of Spanish Cultural Studies, I4, 3 (2013), pp. 29I-306; y Paz Rebollo, María Antonia y Martínez Valerio, Lizette: «Children's programming on Televisión Española under Franco (1958-1975)», European Journal of Communication, volumen 29, 4 (2014), pp. 465-479.

13 Véase nota 5.

${ }_{14}$ El País, 05/07/1980.

is Trilla Bernet, Jaume: La educación fuera de la Escuela, Barcelona, Plantea, 1985. 
M. ${ }^{a}$ CRISTINA HERNÁNDEZ CASTELLÓ

ayuden al portador de esta». Esta petición se debía a que los batidores no iban acompañados por ningún mayor durante sus misiones. En el anverso se incluían las obligaciones de su portador, que estaban encaminadas, por un lado, hacia la difusión del concurso y, por otro, hacia la educación patrimonial, instando a los jóvenes portadores de la misma a formarse en el conocimiento del arte, la historia y la geografía del país.

En el caso de hacer una inscripción de tres o cinco batidores, recibían la denominación de Patrulla.

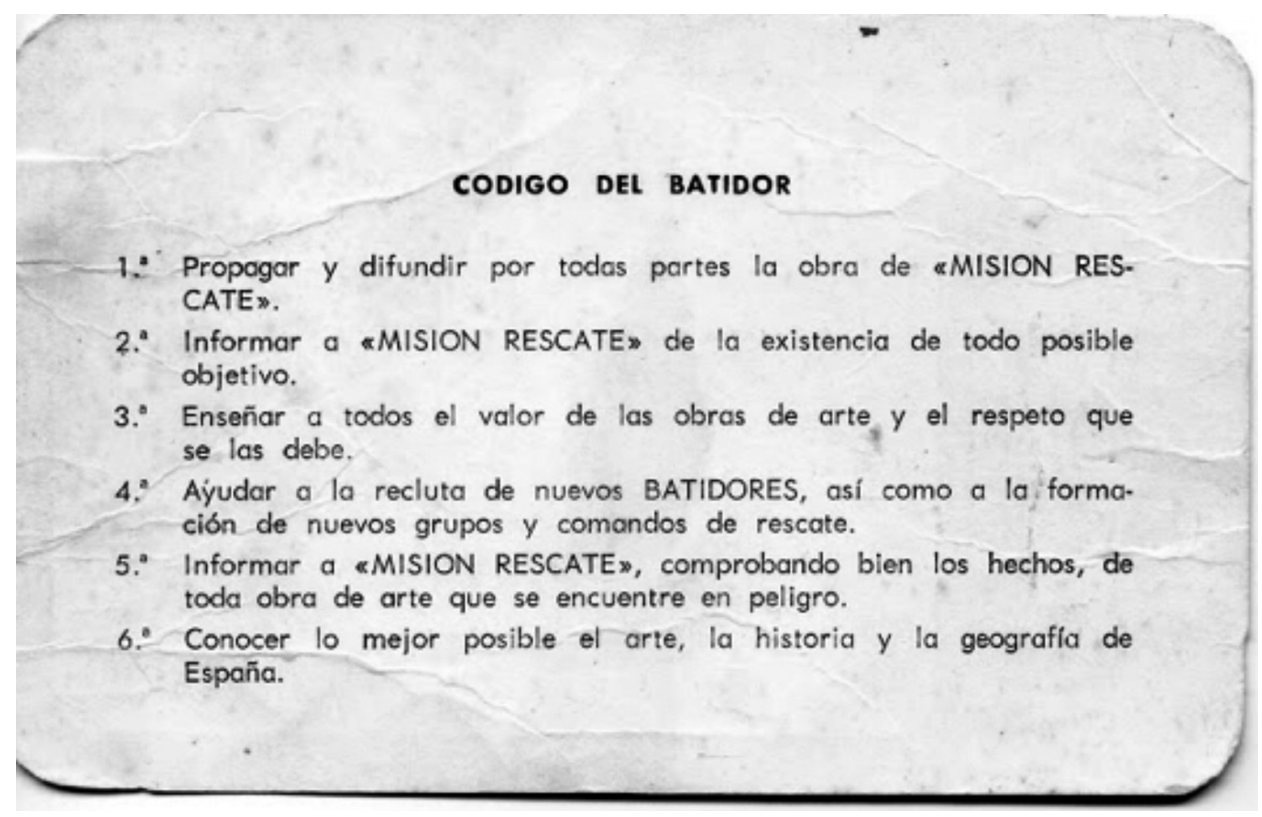

4. Reverso de la credencial de batidor. Archivo fotográfico: familia Cabello Izquierdo.

En las reglas del programa se especificaba que en la solicitud de inscripción debía hacerse mención expresa y detallada del objetivo a rescatar: ruinas históricas, monumentos, imágenes, libros, manuscritos o documentos, cuadros, lugares u objetos históricos. También eran susceptibles de ser rescatados el sitio donde vivió o se alojó circunstancialmente un personaje famoso o cualquier otro lugar en el que hubiese tenido lugar un acontecimiento histórico que, por circunstancias especiales, estuviese olvidado, fuese desconocido o inédito, se hallase desvalorizado o no estuviese catalogado de manera oficial.

Las normas establecían cómo debía desarrollarse la misión una vez fuese autorizada por la dirección del programa, fijando la posibilidad de contar con personas especializadas que pudiesen ayudarles en el proyecto. Nada se dejaba en manos de la improvisación. En el caso de que fuese necesario realizar algún trabajo de excavación, este no podía iniciarse sin contar con la conformidad del 
correspondiente delegado de Excavaciones Arqueológicas, bajo cuyas instrucciones habrían de orientarse todos los trabajos. El permiso se podía obtener de modo directo, o bien a través del programa. En aras de un exhaustivo control de las labores era de obligado cumplimiento el envío de un informe sencillo al programa con una periodicidad mínima bimensual. La documentación remitida podía ir acompañada de material gráfico (fotografías, dibujos, planos...), maquetas e incluso objetos que podían ser utilizados en el programa de TV.

La labor realizada por los concursantes de Misión Rescate fue muy valiosa, recuperando piezas desaparecidas o que no estaban catalogadas. La prensa de la época, tanto nacional como local, se hacía eco de cada nuevo hallazgo ${ }^{16}$. Así el periódico $A B C$ en su edición de la mañana del día 7 de julio de 1968 publicó que gracias al programa se había redescubierto un panteón funerario romano en la zona de Ubrique (Cádiz); un sillar romano desaparecido del arco que precedía al denominado «Puente del diablo» en Martorell (Barcelona), que además fue recolocado en su lugar de origen, y los restos de la necrópolis visigoda de Santa Lucía en Aguilafuente (Segovia), sobre la que profundizaremos más adelante. En esta campaña también fueron premiados el descubrimiento de una inédita Virgen de los Ángeles de «la Roldana», así como el de un San Juan Bautista del xvi y el de un San Fabián de un discípulo de Gregorio Fernández, entre otras esculturas datadas entre los siglos xiv y xviII. Se premió la catalogación de una escultura de «Nuestra Señora de la Luz» como obra del de Diego Copín de Holanda y un cuadro barroco de la Virgen de Sebastián del Llano Valdés ${ }^{17}$. Pero esto no es todo, los hallazgos destacados se sucedían campaña tras campaña, dos años después el grupo de Castrojeriz (Burgos) conseguía la medalla de oro en la iv Campaña por el hallazgo del sepulcro de doña Leonor de Castilla, reina de Aragón, hija de Fernando IV, que se encontraba oculto tras una pared de adobe en la iglesia de Nuestra Señora del Manzano de Castrojeriz ${ }^{18}$. En los premios concedidos en la xv Campaña el trofeo de oro fue para el Colegio Nacional Reina Sofía de Ubrique (Cádiz) por la localización, estudio, catalogación y entrega al Museo Arqueológico de Málaga de un conjunto lítico inédito de i625 piezas de sílex.

El aumento de las colecciones de muchos museos provinciales, e incluso la creación de nuevos espacios expositivos, fue otro de los grandes logros del concurso. Así ocurrió en el caso de Aguilafuente ${ }^{19}$. Misión Rescate traspasó el rango de edad al que estaba destinado, provocando la implicación en muchos casos de gran parte de la sociedad local y propiciando la creación de asociaciones culturales y arqueológicas.

16 Pedimos disculpas al lector por adelantado, pues somos conscientes de que otros muchos sitios arqueológicos y objetos artísticos deberían aparecer en este elenco, sin embargo, por razones obvias de espacio nos limitamos a citar solo aquellos que nos permiten ofrecer un panorama general de la calidad y variedad de piezas artísticas y arqueológicas que abarcaron los proyectos escolares inscritos en Misión Rescate.

${ }^{17} A B C, 07 / 07 / 1968$, p. $6 \mathrm{I}$.

${ }_{18} A B C, 28 / \mathrm{ro} / 1970$, p. 57.

19 Cano López, Santiago: «Misión Rescate. Montoro», Boletín de la Asociación Provincial de Museos locales de Córdoba, n. ${ }^{\circ}$ I4 (2013), p. 424. 


\section{Las excavaciones arqueológicas en Aguilafuente (Segovia): una aproximación histórica}

Hemos señalado al inicio de este trabajo como uno de los sitios arqueológicos que puso en valor Misión Rescate fue el yacimiento de Santa Lucía de Aguilafuente (Segovia). Se trató de un redescubrimiento ya que en la segunda mitad del siglo xIx se había constatado la aparición de un mosaico en la zona.

Las primeras noticias que tenemos sobre los restos romanos de esta localidad son del 4 de marzo de i868 cuando en sesión celebrada en la Diputación Provincial de Segovia los representantes del Partido de Cuéllar notificaron que en Aguilafuente, villa cercana a la localidad cuellarana, se había encontrado un mosaico que «por la muestra que presentaron, es de esperar merezca la consideración del Excmo. Sr. Gobernador [...] ${ }^{{ }^{20}}$. A partir de ese momento y hasta la actualidad se han sucedido en el paraje de Santa Lucía las campañas arqueológicas que han sacado a la luz los principales restos conservados.

El hallazgo había tenido lugar dos días antes de aquella sesión por un vecino del lugar, Bartolomé Ballesteros, quien de forma fortuita encontró un pavimento de mosaico en sus tierras mientras extraía piedras, descubrimiento del que inmediatamente informó a las autoridades del municipio.

Gracias a las actas de las sesiones celebradas aquel año en la Diputación Provincial de Segovia y a los fondos documentales de la Comisión de Antigüedades correspondientes a Castilla y León de la Real Academia de la Historia (RAH), estudiados por Esteban Molina ${ }^{21}$, conocemos los acontecimientos y las decisiones que políticos y eruditos tomaron en los día sucesivos.

Las labores en la primera semana tras al hallazgo fueron frenéticas informándose a la RAH de que tras solo dos jornadas de trabajo la superficie excavada tenía aproximadamente 40 metros cuadrados, lo cual «da una idea del escaso rigor y minuciosidad con el que se estaba interviniendo $»^{22}$. Es más que probable que la escasa profundidad a la que fueron encontrados los restos, aproximadamente un metro, favoreciese esa celeridad. Pero este frenético entusiasmo pronto sufrió una desaceleración llegando a paralizarse por completo los trabajos desde finales de marzo hasta el otoño de ese mismo año, al encontrarse la diputación de Segovia a la espera de obtener los permisos e informes de actuación oportunos por parte de la academia madrileña. Un acontecimiento político

20 Archivo de la Diputación Provincial de Segovia, Acta de la Sesión del 04/03/1868, en: Esteban Molina, Jorge: «El descubrimiento y la excavación arqueológica de los Mosaicos de Aguilafuente en I868 a través de la documentación de la época», Estudios Segovianos, Tomo LII, número Io9 (2010), p. 97.

La historiografía de estas excavaciones ha sido revisada y publicada en varias ocasiones, si bien el estudio más completo es el realizado por el arqueólogo Jorge Esteban Molina, remitiendo a sus trabajos a todo aquel que busque profundizar en el desarrollo histórico de las campañas de este yacimiento. Esteban Molina, Jorge: La villa romana y la necrópolis visigoda de Santa Lucía, Aguilafuente (Segovia). Nuevas aportaciones para su estudio, Segovia, Ayuntamiento de Segovia, 2007 y nota 16.

${ }_{22}$ Ibidem, p. IоI. 
paralizaría de manera definitiva la excavación. Sucedió que la reina Isabel II fue derrocada tras la sublevación militar que se inició en Cádiz el in de septiembre. El levantamiento rápidamente se extendió a toda la geografía española. Los cargos políticos heredados del régimen monárquico incluyendo los locales y provinciales fueron destituidos, de manera que tanto en los ayuntamientos como en las diputaciones provinciales todo trámite administrativo fue inmediatamente paralizado. Una Junta revolucionaria tomó el poder en Segovia el 30 de septiembre instituyendo una nueva corporación provincial que enseguida dio por finalizadas las excavaciones. Poco después, Bartolomé Ballesteros, el dueño del terreno de Santa Lucía, procedió a cubrir los restos y reinició la actividad agrícola sobre el yacimiento.

Sin embargo, en la memoria de los habitantes de Aguilafuente el recuerdo del mosaico descubierto siguió vivo y así en mayo de I897 el profesor de instrucción pública y titular de la escuela de niños de Aguilafuente Francisco Gómez Braña informó por carta a la Real Academia de la Historia de la existencia de aquel yacimiento, reclamando la atención de la institución con la intención de que se reactivasen los trabajos de excavación que por décadas habían sido suspendidos. El vacío documental tras esta petición lleva a Esteban Molina a pensar que la solicitud no tuvo respuesta, cayendo en saco roto los intentos del maestro de reanudar los trabajos ${ }^{23}$.

\section{El grupo de rescate $n .^{\circ}$ 349: una nueva oportunidad para el yacimiento de Santa Lucía}

La fortuna de este yacimiento cambió gracias a la emisión del programa $M i$ sión Rescate. En la Escuela Graduada de niños de Aguilafuente se formó el Grupo de Rescate n. ${ }^{\circ} 349$ que, bajo la dirección de su maestro don José Martín Pascual, jugó un papel decisivo en el afloramiento de los mosaicos romanos de la villa y en el descubrimiento de la ubicación de la necrópolis visigoda.

Participaron por primera vez en la campaña que tuvo lugar en 1967-1968, noticia que $A B C$ publicó a principios de $1968^{24}$. El mismo periódico publicaba una fotografía de los redescubiertos mosaicos, señalando a pie de página que los pavimentos hallados presentaban decoraciones a base de abstracciones florales y motivos geométricos ${ }^{25}$. Tan solo cuatro días después el terreno excavado había aumentado considerablemente y ya se describía el hallazgo como un pavimento cuyas teselas dibujaban cuadros, cada uno de los cuales tenía un motivo ornamental distinto. En el centro destacaban unos caballos deteriorados -que al

23 Ibídem, pp. II8-I2I.

${ }^{24} A B C, 2 \mathrm{I} / \mathrm{oI} / \mathrm{I} 968$, p. 62.

${ }_{25} A B C, 17 / 0 I / 1968$, p. I7. A pesar de la escasa calidad de la imagen, resulta relevante la presencia de un grupo de niños al fondo de esta y de un hombre unos pasos por delante de ellos, posiblemente se tratasen de los miembros del equipo 349 de Aguilafuente y su maestro, que asistían a la visita de las autoridades, a quienes podemos ver situados en primer término a la derecha. 


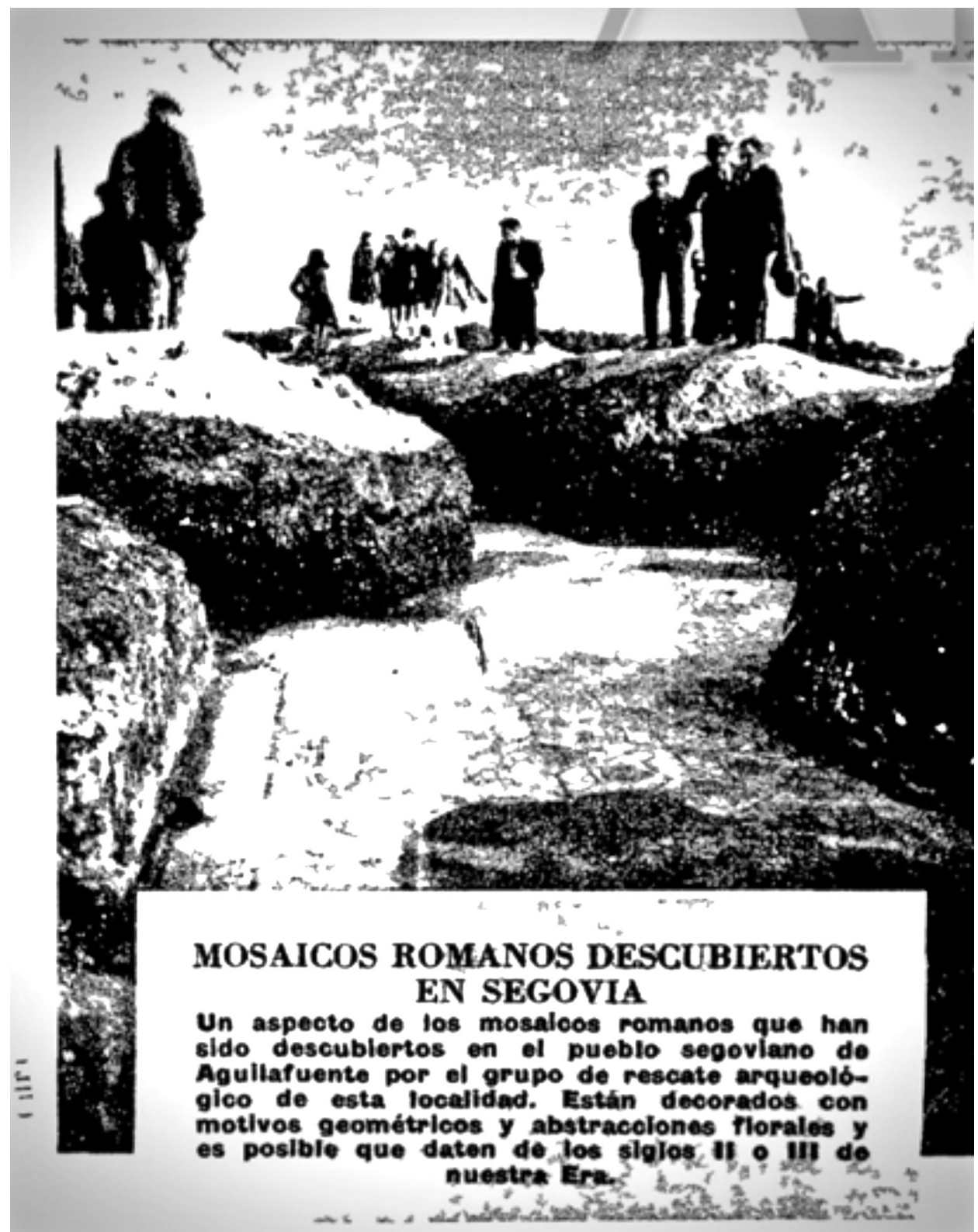

5. Fotografía publicada en el $A B C$ del 17 de enero de 1968.

parecer ya lo estaban en I868- y las palabras «Eufrata»y «Tagus». Se advertía de la existencia en otro sector próximo de mosaicos, con dibujos geométricos, y se ponía en conocimiento de los lectores de la exhumación en la misma zona de restos arquitectónicos, de un pavimento que pudiera ser una calzada y de restos 


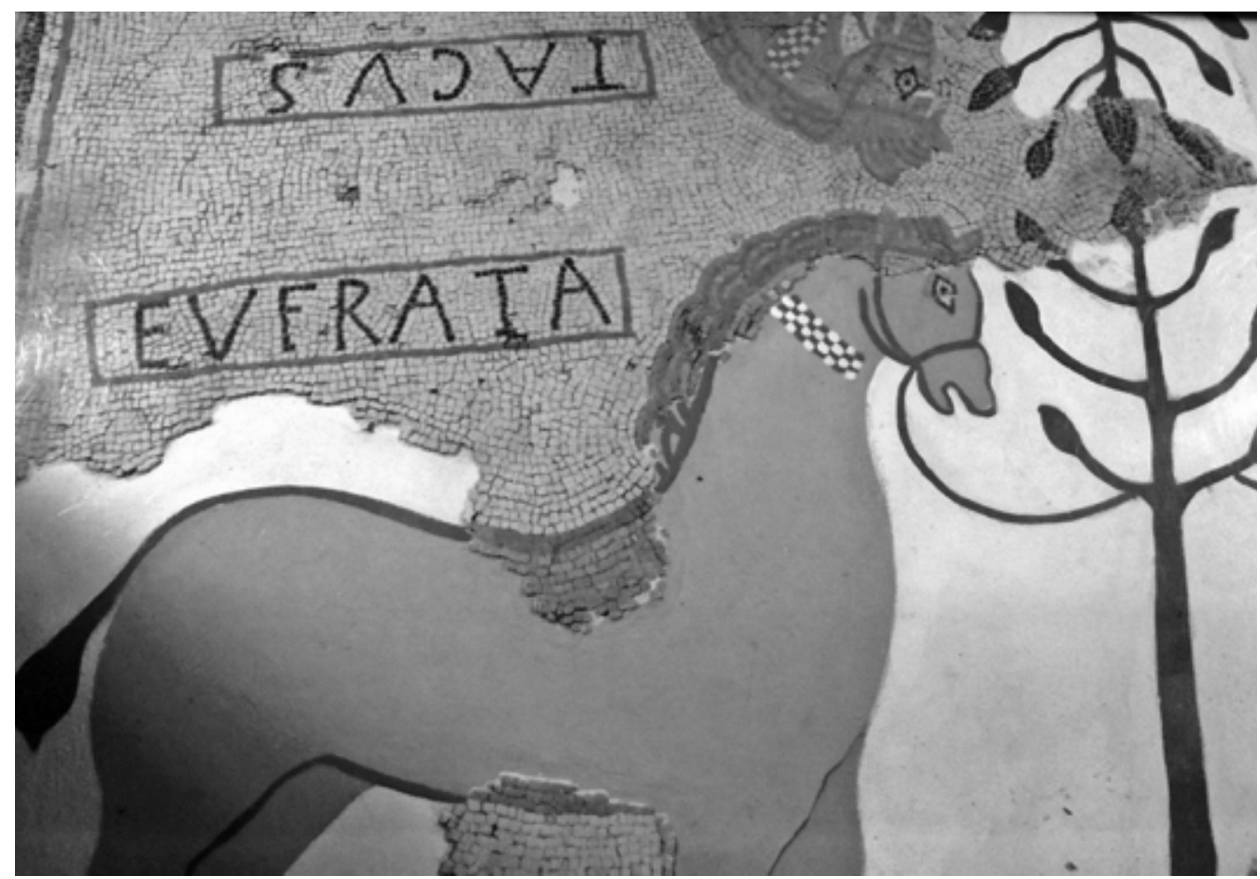

6. Detalle del mosaico de los caballos de Aguilafuente (Segovia). Autora: Laura Frías Alonso.

humanos. También La Vanguardia se hacía eco del hallazgo ampliando la información con la noticia de la reciente visita del secretario técnico del Instituto Central de Restauraciones de Obras de Arte y Arqueología junto a otro especialista, cuyo nombre no aparece en la citada publicación, y junto al delegado provincial de Bellas Artes ${ }^{26}$.

En el mes de marzo el grupo de Aguilafuente recibió uno de los trofeos de plata de la segunda campaña de Misión Rescate por los valiosos descubrimientos que hasta el momento habían realizado. Tal era la calidad de lo encontrado que al parecer alguna voz se había alzado en las semanas previas manifestando la intención de llevarlos a la capital. Ante la incertidumbre que mostraba el Ayuntamiento de la pequeña localidad segoviana por el posible traslado de los mosaicos a finales del mes de marzo el director de Bellas Artes se vio obligado a desmentir aquellos rumores, con la consiguiente satisfacción por parte de la corporación municipal ${ }^{27}$.

El día 28 de ese mismo mes se anunciaban nuevos descubrimientos esta vez con la intervención del personal del Instituto Nacional de Restauración y de la Dirección General de Bellas Artes, pues dada la importancia de los hallazgos anteriores y siguiendo las bases del concurso era obligatoria la intervención de

26 La Vanguardia Española, 26/or/1968, p. 9.

$27 A B C, 20 / 03 / 1968$, p. 67. 
especialistas, eso sí, siempre contando con la ayuda de los alumnos. A partir de ese momento las excavaciones estuvieron dirigidas por don Vicente Viñas ${ }^{28}$, a quien se unió un año después Rosario Lucas ${ }^{29}$.

De nuevo el $A B C$ informaba sobre el asunto:

Se ha excavado ya una villa romana. Los muros de cuyas habitaciones aparecen, en algún caso, adornados con estucos. Igualmente se han hallado otros mosaicos, restos de cerámica, monedas de diversas épocas y un capitel de columna de orden corintio de gran interés. También han aparecido restos de una antigua ermita, que se cree fue la de Santa Lucía, y sobre los restos de la propia villa romana, en la zona de Poniente, ha surgido de las excavaciones una necrópolis visigótica, de marcada importancia. Las sepulturas de esta son femeninas en su mayor parte y aparece en ellas el ajuar correspondiente: fíbulas, broches de cinturón. collares, etc..$^{30}$.

En I992, gracias a la Junta de Castilla y León, se limpió el yacimiento y se consolidaron los muros, nuevas intervenciones se están realizando en el $2018^{35}$.

Contemporáneamente a las excavaciones se impartieron conferencias por parte de los arqueólogos encargados del proyecto, y se realizaron exposiciones y proyecciones de documentales, en definitiva, se amplió el proyecto de la misión de rescate a la misión cultural con un claro enfoque divulgativo.

Los premios y trofeos finales de la segunda campaña de Misión Rescate se fallaron en el verano de ese mismo año. En aquella ocasión el grupo de rescate 349 fue galardonado con el Trofeo de Oro por la localización exacta y afloramiento de los valiosos mosaicos romanos que ya les habían hecho valedores de un trofeo de plata. Se especificaba en la publicación que el valioso trabajo llevado a cabo por los jóvenes había permitido a los expertos de Bellas Artes descubrir también una necrópolis visigoda en cuyas tareas auxiliares habían colaborado.

En paralelo a las excavaciones y a los eventos científicos y culturales que surgieron alrededor, se fraguaba en Aguilafuente la idea de contar con un lugar adecuado para conservar y exponer todo el material que iba encontrándose en Santa Lucía. Ya en el mes de octubre de 1968 el alcalde de la localidad solicitaba 50.000 pesetas a la Dirección General de Bellas Artes para la creación de un lugar donde exponer los objetos encontrados en las excavaciones de Santa Lucía. Meses

${ }_{28}$ Vicente Viñas ha pasado a la historia como uno de los más brillantes conservadores y restauradores del patrimonio en España. En una primera etapa se dedicó a la restauración arqueológica para decantarse, finalmente, por la restauración de documentos gráficos, materia en la que fue pionero.

29 Las excavaciones arqueológicas de los profesores Viñas y Lucas se prolongaron desde 1968 hasta 1972, momento en el que los trabajos se paralizaron. Sus trabajos fueron difundidos a través de congresos y publicaciones científicas, como LucAs, M. ${ }^{a}$ Rosario y ViÑAs, Vicente: «La villa romana de Aguilafuente (Segovia)», en Symposium Internacional «Segovia y la Arqueología Romana», Universidad de Barcelona, 1977, pp. 239-255 y LuCAs, M. ${ }^{a}$ Rosario y VIÑAs, Vicente: «Nuevos mosaicos romanos y otros hallazgos arqueológicos en la provincia de Segovia», Estudios Segovianos, tomo XXIII (I97I), pp. 7I-IO4.

30 $A B C, 28 / 03 / 1968$, p. $67-68$.

${ }_{31}$ El Norte de Castilla, or/o5/2017, en: http://www.elnortedecastilla.es/segovia/201705/or/aguilafuente-rehabilitara-yacimiento-santa-20170430144950.html (consultado el 22/12/2017). 
después el Ayuntamiento contemplaba la posibilidad de adquirir la iglesia de San Juan, por entonces en manos privadas y convertida en un granero, con la intención de convertirla en aula arqueológica para las piezas encontradas. Sin embargo, la compra no se llevó a cabo ante la negativa de la Caja de Ahorros y Monte de Piedad de Segovia de financiar las obras de restauración ${ }^{32}$. Cuando a principios de 1969 aparecieron más mosaicos, los medios se hicieron eco de la intención de crear en la localidad un museo arqueológico con los restos romanos y visigodos encontrados ${ }^{33}$. Sin embargo, el proyecto tuvo que esperar hasta que en noviembre de 1989 el Consistorio se hizo con la propiedad de la iglesia de San Juan para ubicar en ella el ansiado museo. La restauración del edificio se dilató en el tiempo, y once años después, la Junta de Castilla y León y el Ayuntamiento de Aguilafuente firmaron el 2 de octubre del año 2000 el convenio de colaboración para la creación del Aula Arqueológica ${ }^{34}$ cuya inauguración tuvo lugar el 26 de octubre de $200 \mathrm{I}^{35}$.

\section{A modo de conclusión}

Hoy en día sería impensable que los jóvenes emprendiesen remociones de tierra en aquellos lugares donde se hubiese producido un hallazgo casual de objetos arqueológicos que no hubiera sido comunicado inmediatamente a la Administración competente, o en aquellos lugares donde se presumiese la existencia de yacimientos o restos arqueológicos ya que la ley de Patrimonio Histórico español I6/1985 prohíbe este tipo de prácticas.

Tampoco sería factible que un grupo escolar rebuscase en un archivo documentos no catalogados, o que registrase en una iglesia cada recoveco a la espera de encontrar una obra artística inédita. Sin embargo, debemos situar en su contexto este concurso, un momento en el que tanto a través de la educación formal como de la educación informal se pretendía diseñar una nueva identidad a los españoles en la que se proyectasen «principios e ideales rectores de la recuperación nacional» ${ }^{36}$.

El valor educativo del programa residió en el papel que jugó para que las distintas localidades comprendiesen su entorno histórico artístico y se reconociesen en el mismo, creando una identidad grupal. Asimismo, contribuyó a desarrollar una actitud de respeto y conservación hacia el Patrimonio. Misión Rescate contribuyó a una mejora cualitativa en la educación patrimonial de la época. Tal y como la profesora Roser Juanola (2005) argumenta, el conocimiento del patrimonio

32 Una breve aproximación a la historia del aula arqueológica de Aguilafuente en: Frías Alonso, Laura: «El aula arqueológica de Aguilafuente (Segovia): creación de un museo», Boletín del Museo Arqueológico Nacional, 35 (2017), pp. II44-II47.

33 La Vanguardia Española, i5/03/1969, p. II.

34 BOCyL, n. ${ }^{\circ}$ 191, del lunes 2 de octubre de 2000, p. I2128. Publicación del convenio de colaboración entre la Junta de Castilla y León y el Ayuntamiento de Aguilafuente (Segovia) para la difusión social de la villa romana, a través de la creación de un aula arqueológica.

35 El Norte de Castilla, 23/12/2017 en: http://www.elnortedecastilla.es/segovia/honorse-apoyara-consolidacion-20I7I223I208II-nt.html (consultado el 24/12/2017).

${ }_{36}$ Fontana, Josep: Enseñar Historia con una guerra de por medio, Barcelona, 1999, p. 54. 
contribuye a fomentar la percepción y expresión de emociones propias y opiniones, y de igual modo ayuda a la construcción de un conocimiento interdisciplinar: histórico, social, natural y cultural.

Conocer la organización y el desarrollo que tuvo el programa nos ha permitido tomar conciencia de varias cuestiones referentes a la educación patrimonial y al papel de los mass media en la misma.

I. Como ha defendido la profesora Fontal, «el patrimonio es la relación entre bienes y personas» ${ }^{37}$, es por tanto incuestionable que el programa Misión Rescate contribuyó a reavivar la vinculación entre los bienes culturales y las personas, o, lo que es lo mismo, entre el objeto-sujeto, que tan necesaria era en la España del tardofranquismo, guiando a sus jóvenes concursantes a través del camino de la educación patrimonial mediante microproyectos de redescubrimiento y puesta en valor de bienes artísticos y yacimientos arqueológicos de su entorno.

2. Se consiguió una vinculación estrecha entre emoción y patrimonio. Al generar experiencias en torno al patrimonio local, los jóvenes rescatadores se convirtieron en agentes activos de su valoración y conservación.

3. Esa implicación de los niños y adolescentes de cada localidad traspasó el ámbito escolar y se propagó por toda la comunidad, al tomar conciencia del valor de los objetos artísticos y yacimientos que atesoraban en sus territorios.

4. Los mass media contribuyeron poderosamente por entonces, y contribuirían en la misma medida o más en la actualidad, a generar conciencia de respeto y valoración hacia la cultura material de un país.

5. La difusión del programa a nivel nacional contribuyó a crear riqueza económica en aquellos lugares en los que actuaron las patrullas de rescate.

37 Fontal Merillas, Olaia (coord.): La educación patrimonial. Del patrimonio a las personas, Gijón, 2013, p. I8. 
